\title{
Protein velocity and acceleration from single-cell multiomics experiments
}

\author{
Gennady Gorin ${ }^{1}$, Valentine Svensson ${ }^{2^{*}}$ and Lior Pachter ${ }^{2^{*}}$ (D)
}

\begin{abstract}
The simultaneous quantification of protein and RNA makes possible the inference of past, present, and future cell states from single experimental snapshots. To enable such temporal analysis from multimodal single-cell experiments, we introduce an extension of the RNA velocity method that leverages estimates of unprocessed transcript and protein abundances to extrapolate cell states. We apply the model to six datasets and demonstrate consistency among cell landscapes and phase portraits. The analysis software is available as the protaccel Python package.
\end{abstract}

Keywords: Protein acceleration, Protein velocity, RNA velocity, Transcriptomics, Multiomics, Bioinformatics, Computational biology

\section{Background}

Recent technological innovations that allow for assaying multiple modes of cell states at single-cell resolution are creating opportunities for more detailed biophysical modeling of the molecular biology of cells. Specifically, genome-wide probing of molecular states is revealing detailed information about the functional diversity of cells as determined by gene regulation, transcription, processing, and translation. The ability to probe cell states has been driven by improvements in single-cell RNA sequencing (scRNA-seq) methods [1] and advances in multiomics [2]. These methods allow researchers to quantify mRNA and protein expression levels in individual cells [3-5]. Furthermore, scRNA-seq can discriminate between nascent and processed transcripts. The recently described RNA velocity [6] method takes advantage of this feature of single-cell RNA-seq to fit a first-order system of ordinary differential equations describing gene-specific splicing [7] and to infer kinetic trajectories of single cells.

RNA velocity exploits the transfer of information in gene expression to extrapolate future cell states. In brief, the current population of unspliced transcripts is slated to be processed (Fig. 1a) and thus contains information regarding the future population of spliced transcripts. We extend this

\footnotetext{
*Correspondence: valentine@nxn.se; Ipachter@caltech.edu

${ }^{2}$ Division of Biology and Biological Engineering \& Department of Computing and Mathematical Sciences, California Institute of Technology, Pasadena, USA Full list of author information is available at the end of the article
}

logic as follows [8]: the current population of proteins was translated from spliced RNA and thus contains information regarding the past population of spliced transcripts (Fig. 1b). We extend the RNA velocity model to protein translation, resulting in an analogous mathematical formulation for protein count extrapolation (Fig. 1a). We emphasize that unlike methods that require time-series measurements [9-11], our method estimates protein translation kinetics from a single time-point. To visualize the apparently disparate RNA and protein estimates in a single cell state representation, we adapt a method [6] for embedding dynamical information based on a distance metric in a high-dimensional space (Fig. 1c). We provide the Python package protaccel to facilitate analysis, and apply it to datasets to estimate their past and future cell states and identify trends in acceleration behavior.

\section{Results and discussion}

We analyze six peripheral blood mononuclear cell (PBMC) datasets, collected using four different technologies. The dataset metadata is outlined in Table 1 . The four technologies are CITE-seq [3], REAP-seq [4], ECCITE-seq [5] (with two datasets: "ctrl," a healthy control, and "CTCL," a cutaneous $\mathrm{T}$ cell lymphoma patient), and 10X Genomics feature barcoding (with two datasets: "1k" and "10k" cells).

The approximately linear spliced RNA/protein phase plots (Additional file 1: Figures S1-S6) are qualitatively consistent with the first-order and constant-parameter 
a

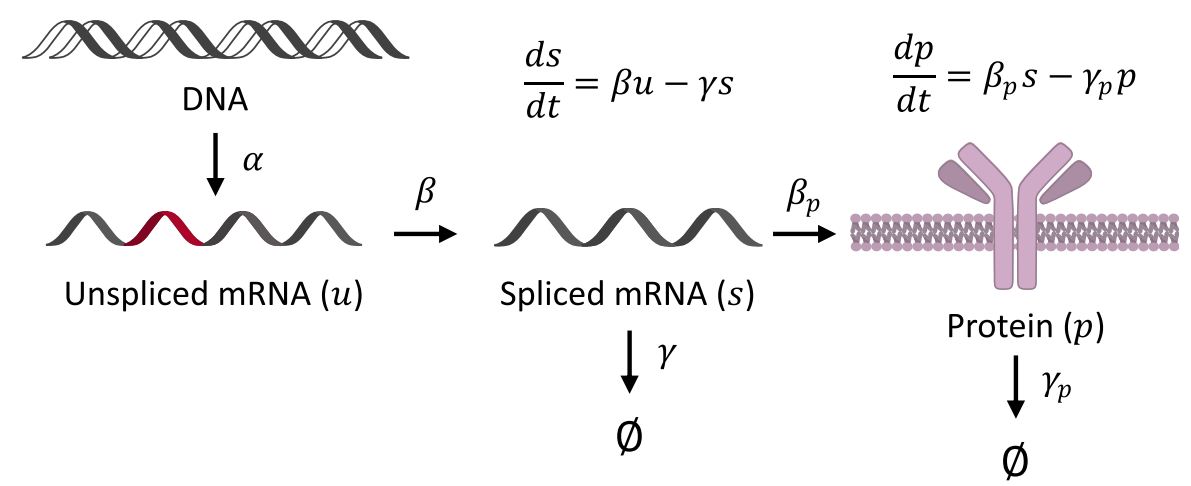

b

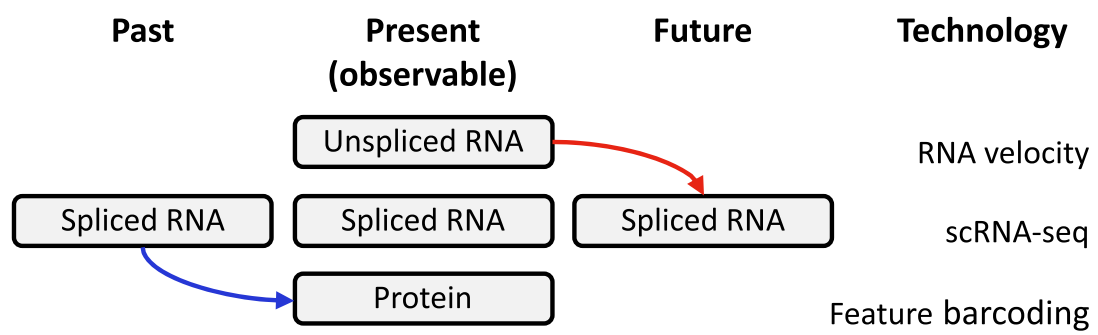

C
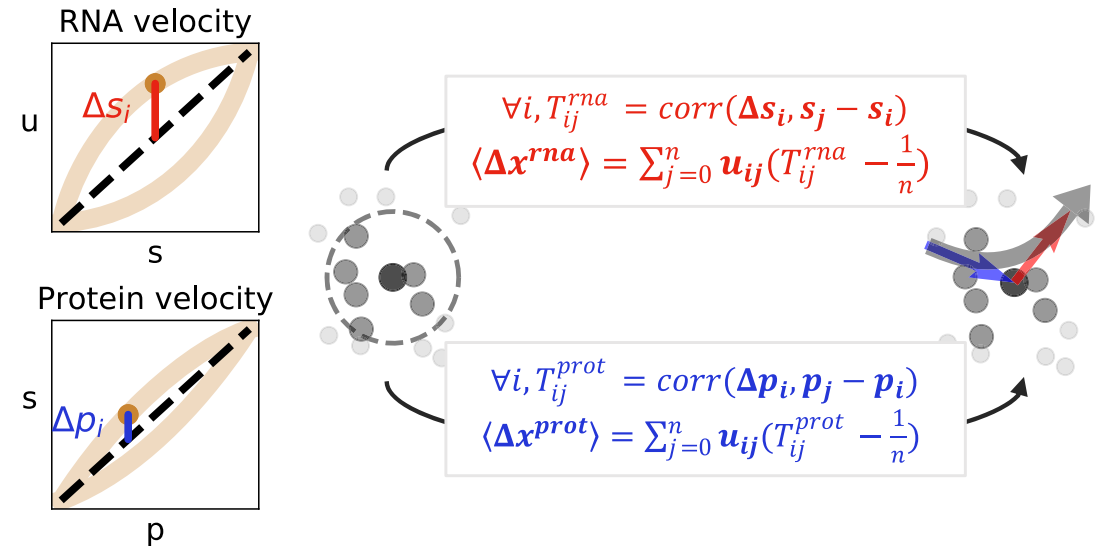

Fig. 1 Model structure and parameter inference. a A single gene's information transfer through transcription, splicing, and translation, and the ordinary differential equations governing the spliced mRNA and protein populations. $\mathbf{b}$ Conceptual framework for extrapolation from snapshot sequencing data. c Protein acceleration workflow: estimation of equilibrium states $u=\gamma s$ and $s=\gamma_{p} p$ (black dashed lines) from imputed genespecific population data (light brown), gene-specific extrapolation to calculate $\Delta s_{i}$ and $\Delta p_{i}$, identification of nearest neighbors (dark gray: cell $i$, intermediate gray: $n$ neighboring cells $j$, light gray: non-neighbor cells, circle: neighborhood), calculation of transition probabilities and embedded velocities (red: RNA velocity, blue: protein velocity, $T_{i j}$ : transition probability from cell $i$ to neighbor $j$, $u_{i j}$ : unit vector from cell $i$ to neighbor $j$ ), and visualization of acceleration (blue arrow: protein velocity, red arrow: RNA velocity, combined curvature: gray Bézier curve)

model of protein production, although we do observe some deviations by cell type. A subset of linear gene/ protein pairs (Additional file 1: Table S1), manually selected from the phase plots according to concordance with the model, was used to estimate the gene-specific protein velocities. To calculate RNA velocity, we use a broad panel of genes with robust unspliced detection, high variation, and good agreement with the ODE model (sample genes and fits shown in Additional file 1: Figures S7-S12). We extrapolated the cell states, then embedded them in a projection calculated from the spliced mRNA space (Additional file 1: Supplementary Note).

The cell type-specific RNA velocities (Additional file 1: Figure S13-S18) depict a highly directional landscape. The corresponding protein velocities (Additional file 1: Figures S19-S24) are rather noisier as a result of sparser 
Table 1 Protein acceleration datasets and parameters

\begin{tabular}{|c|c|c|c|c|c|c|}
\hline Dataset & CITE-seq & REAP-seq & ECCITE-seq ctrl & ECCITE-seq CTCL & $10 \times 1 \mathrm{k}$ & $10 \times 10 k$ \\
\hline RNA data & GSM2695381 & GSM2685238 & GSM3596095 & GSM3596100 & See Methods & See Methods \\
\hline Protein data & GSM2695382 & GSM2685243 & GSM3596096 & GSM3596101 & See Methods & See Methods \\
\hline Alignment software & Cell Ranger 2.2 & Cell Ranger 1.3 & Cell Ranger 3.0 & Cell Ranger 3.0 & kallisto 0.46 & kallisto 0.46 \\
\hline Counting software & velocyto 0.17 & velocyto 0.17 & velocyto 0.17 & velocyto 0.17 & kallisto 0.46 & kallisto 0.46 \\
\hline Reference genome & GRCh38 & hg19 & hg19 & hg19 & GRCh38 & GRCh38 \\
\hline Cell count & 1780 & 3158 & 5084 & 5317 & 709 & 7855 \\
\hline Velocity genes & 1172 & 1338 & 591 & 667 & 1114 & 920 \\
\hline Antibodies & 10 & 41 & 49 & 49 & 17 & 17 \\
\hline Velocity proteins & 7 & 16 & 11 & 12 & 7 & 8 \\
\hline Cell types found & 5 & 4 & 4 & 3 & 5 & 5 \\
\hline Imputation $k$ & 400 & 800 & 800 & 800 & 50 & 50 \\
\hline Clustering method & MVP & RVP & RVP & MVP & MVP & MVP \\
\hline Embedding & $\mathrm{PC} 2 / 3$ and $\mathrm{t}-\mathrm{SNE}$ & $\mathrm{t}-\mathrm{SNE}$ & $\mathrm{t}-\mathrm{SNE}$ & $\mathrm{t}-\mathrm{SNE}$ & $\mathrm{t}-\mathrm{SNE}$ & t-SNE \\
\hline
\end{tabular}

MVP ModularityVertexPartition, RVP RBERVertexPartition, PCA principal component, $t$-SNE t-Stochastic Neighbor Embedding

data collection (dozens of proteins vs. thousands of genes). We used a Gaussian kernel to determine the net velocities at regular grid points. The RNA and protein velocity fields (Additional file 1: Figures S25-S30) suggest that alignment between the two is strongly associated with cell type. The combination of RNA and protein velocities reveals the curvature of the cell state landscape. In a conceptual sense corresponding to Fig. 1b, the immediate protein velocity and the underlying RNA velocity yield a second-order estimate of protein acceleration driven by upstream unspliced mRNA modulation. We visualize cell movement in the embedding using a Bézier curve calculated from three points corresponding to past, present, and future cell states. A high-curvature Bézier curve corresponds to high acceleration.

The protein acceleration landscapes show a diversity of dynamics identifiable across datasets (Fig. 2a, Additional file 1: Figure S31-S36). A distinct set of B cells has high acceleration (CITE, REAP, 10X 1k, 10X 10k); another set, which forms a separate cluster in t-SNE, has low acceleration (CITE, REAP, 10X 10k). T lymphocytes tend to show low acceleration and low mobility in general (all datasets), although a mobile subset is occasionally seen and forms a cluster with mobile monocytes (CITE, REAP, possibly $10 \mathrm{X} 1 \mathrm{k}$, possibly 10X 10k). Monocytes are mobile and exhibit a mixture of unidirectional and accelerated dynamics in a single cluster (CITE, REAP, ECCITE ctrl, 10X 1k, 10X 10k).

We found that subsets of B cells and of T lymphocytes exhibit strong protein acceleration. We hypothesize that the $\mathrm{B}$ cell partitioning corresponds to the differences between cell subtypes, e.g., mature B cells, which are resting [12] and require dedicated $\mathrm{T}$ cell activation [13], and plasma cells, which quickly respond to stimuli [14] and would be expected to have high acceleration on the relevant timescales. The $\mathrm{T}$ lymphocyte behavior may reflect recent findings that describe mRNA transcript "pile-up" due to heavily suppressed translation in naïve CD4+ T cells [15], or potential lymphocyte plasticity [16]. The monocyte behavior may correspond to the steady-state partitioning between monocyte subtypes [17], such as the transition from classical to non-classical circulating monocytes [18]. However, due to the imperfect separation of cell types in the embedding, we caution against over-interpretation of aggregated velocities.

The quality of data between the four different technologies is quite disparate. CITE-seq and 10X technologies appear to give strong velocity signals; inspection of raw phase portraits suggests that the results are fairly reliable (Additional file 1: Figures S1, S5-S7, S11-S12). REAP-seq yields lower RNA counts (Additional file 1: Figures S2, S8) and noisier dynamics. Finally, ECCITE-seq yields extremely sparse acceleration landscapes (Additional file 1: Figures S33-S34), which result from the very shallow sequencing of spliced transcripts: we confirmed that ECCITE-seq captures 1-2 orders of magnitude fewer RNA molecules per cell than CITE-seq or REAPseq, which is consistent with Fig. 1b of Mimitou et al. [5] (Additional file 1: Figure S37). Comparison to unfiltered pseudoaligned 10X data shows that the ECCITE-seq RNA counts are roughly comparable to counts in empty droplets in the 10X feature barcoding technology. Overall, the CITEseq and feature barcoding technologies appear to be by far the most reliable.

In addition to using genes with linear behavior to infer velocity, we qualitatively confirmed consistency between datasets for the gene CD4, which has a striking non-linear appearance (Fig. 2b). We hypothesize that the non-linear behavior corresponds to regulatory differences due to cell type; in the context of our model, the data seem to suggest a unique, low degradation rate in CD4+ $\mathrm{T}$ lymphocytes and a different, high degradation rate in all other blood cell types. 
a

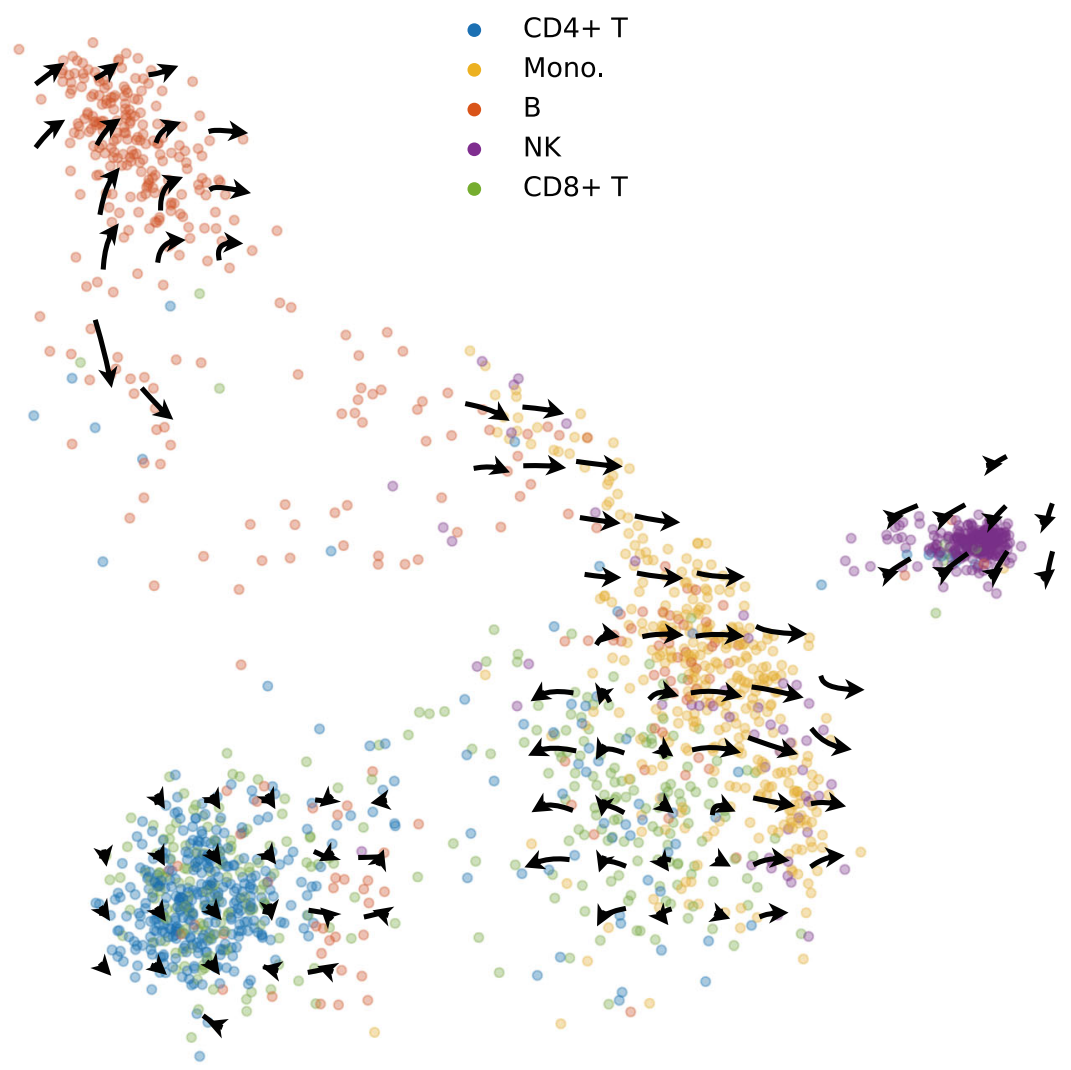

b

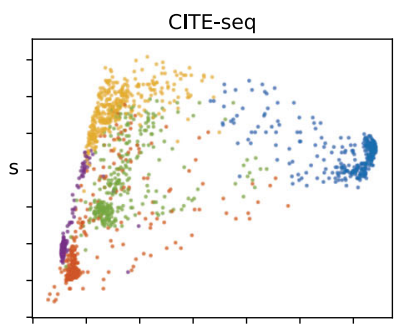

$\mathrm{p}$

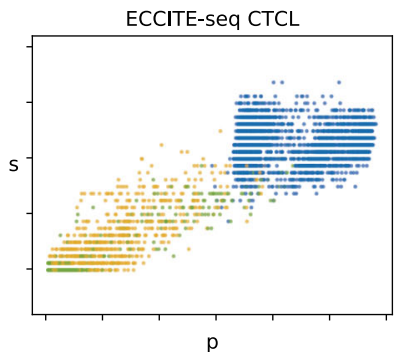

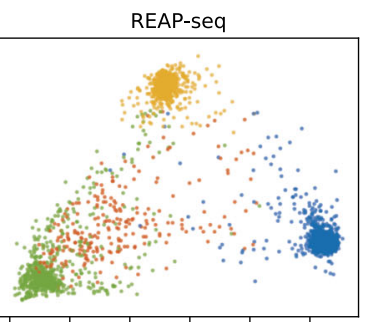

$\mathrm{p}$

10X 1k

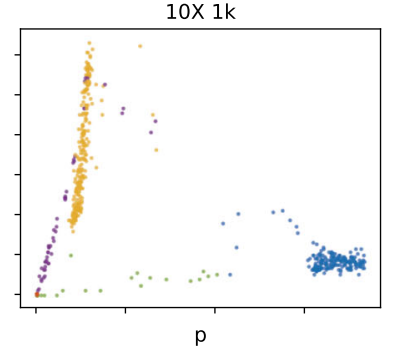

ECCITE-seq ctrl

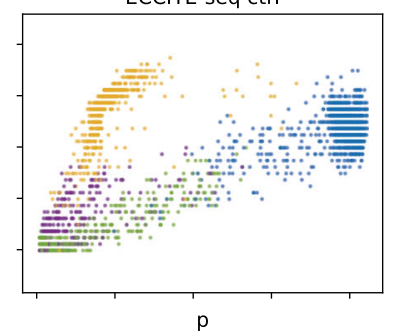

10X 10k

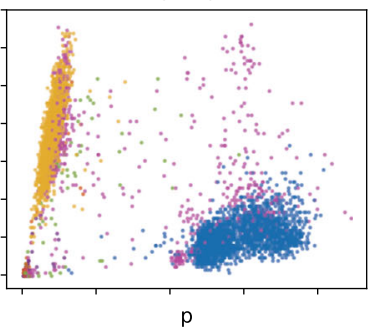

Fig. 2 Protein acceleration visualization. a CITE-seq PBMC protein acceleration, visualized on a grid in principal component space. b Spliced RNA/ protein phase portraits of CD4 in six PBMC datasets. Dot color identifies cell type (blue: CD4+ T, red: B, yellow: monocytes, green: CD8+ T, purple: natural killer, pink: not identifiable unambiguously)

Our qualitative protein acceleration framework does not attempt to account for regulatory differences between cell types. Future work may involve more granular models to enable inference of local rather than global parameters, e.g., the determination of separate parameters for the
CD4+ T lymphocytes and other cell types for the CD4 gene (Fig. 2b). Current protein quantification protocols are adapted for histological markers on the cell surface; technology that can quantify cytosolic protein could aid in more extensive studies of cell dynamics and open a 
broader range of investigations tractable by protein acceleration, as discussed in Additional file 1: Supplementary Note. In particular, we anticipate this method is naturally applicable to inferring and validating cell state vector fields [19]. Finally, the simultaneous quantification of mRNA and regulatory cytosolic proteins would greatly aid in the implementation of physically realistic models of gene expression which explicitly account for regulation by observed transcription factors.

\section{Methods}

The key metadata, physiology, and parameters used for the six datasets are summarized in Table 1. GSM numbers correspond to Gene Expression Omnibus (GEO) samples. 10X Genomics PBMC datasets are available at the 10X Genomics website [20, 21]. Aligned sequence files released alongside each original publication were used whenever available. The velocyto 0.17 commandline interface was used to generate unspliced count matrices for the CITE-seq, REAP-seq, and ECCITE-seq datasets; kallisto 0.46 was used for the 10X datasets.

The velocity calculation and visualization processes are described in detail in Additional file 1: Supplementary Note. In brief, scRNA-seq and feature barcoding data were smoothed using a nearest-neighbor connectivity matrix, generated using the scikit-learn 0.20.0 Python package [22]. For each cell, unspliced RNA, spliced RNA, and protein counts were replaced with the mean value of $k$ neighbor cells. For ease of visualization, Louvain clustering was performed using the louvain 0.6.1 Python package [23]. Cell types were manually assigned based on markers (Additional file 1: Table S2) reported in CITE-seq and REAP-seq publications [3, 4] (Additional file 1: Figures S38-S43).

We implemented the protein acceleration workflow as the protaccel Python package [24]. protaccel 0.2 was used for all analyses in this article, with the exception of Additional file 1: Figures S52-S54, which used protaccel 0.301. To calculate RNA velocities, we fit extreme quantiles of the imputed spliced/unspliced RNA phase plots, filtered to select "velocity genes" with phase plots described sufficiently well by the linear fit $\left(R^{2}>0.1\right)$, estimated the spliced RNA degradation rates, then calculated deviations from the equilibrium line. To calculate protein velocities, we followed the same process, albeit using protein/spliced RNA phase plots and manually selecting "velocity proteins" with qualitatively linear phase plot appearance.

To visualize the velocities, we generated low-dimensional embeddings for the cells, selected to be a set of principal components (PCs) for CITE-seq and a t-Stochastic Neighbor Embedding (t-SNE) based on the top 25 PCs for all other datasets, as well as CITE-seq in Additional file 1: Figure S31 [25]. The PC and t-SNE calculations were performed using the scikit-learn 0.20.0 Python package [22]. Consistently with the original RNA velocity publication [6], we assumed the net velocity direction can be represented on a low-dimensional embedding by calculating transition probabilities to an embedding neighborhood of 500 cells. We computed these transition probabilities by calculating the correlation between high-dimensional velocity and directions to the embedding neighbors, both processed with a variance-stabilizing square root transformation. The high-dimensional space of the RNA velocity workflow is the space of velocity genes; the corresponding high-dimensional space of the protein velocity workflow is the space of velocity proteins. Each embedding was partitioned into a $20 \times 20$ point grid, representing cell states at $t_{0}$; grid arrows were generated by applying a Gaussian kernel (smoothing parameter $\sigma=0.5$ ) to the cell-specific velocities of 200 cells nearest the grid point. The forward extrapolation of each grid point, corresponding to information about $t_{+1}$ inferred from RNA velocity, was calculated by adding the aggregated RNA velocity vector to the grid point vector. The backward extrapolation of each grid point, corresponding to information about $t_{-1}$ inferred from protein velocity, was calculated by subtracting the aggregated protein velocity vector from the grid point vector. We produced curved arrows corresponding to the entire trajectory by fitting a second-order Bézier curve to each grid point's $t_{-1}, t_{0}$, and $t_{+1}$ locations. The fit was performed using the bezier 0.9.0 Python package.

We performed all simulations using MathWorks MATLAB 2018a.

Scripts to reproduce the results of this paper are available at GitHub [26]. Raw datasets for protein acceleration analysis (velocyto loom files with mRNA counts and csv files with protein counts) are available on figshare [27-30].

\section{Supplementary information}

Supplementary information accompanies this paper at https://doi.org/10. 1186/s13059-020-1945-3.

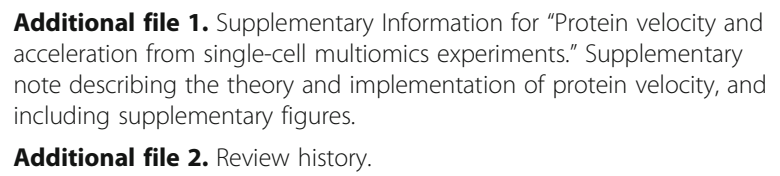

Additional file 1. Supplementary Information for "Protein velocity and acceleration from single-cell multiomics experiments." Supplementary note describing the theory and implementation of protein velocity, and including supplementary figures.

Additional file 2. Review history.

\section{Acknowledgements}

We thank the authors of Mimitou et al. [5] for providing velocyto pipeline outputs for ECCITE-seq datasets.

Review history

The review history is available as Additional file 2.

Peer review information

Barbara Cheifet was the primary editor of this article and managed its editorial process and peer review in collaboration with the rest of the editorial team. 


\section{Authors' contributions}

GG implemented the method and performed the analysis under the supervision of VS. VS and LP developed the protein acceleration extension of RNA velocity. GG, VS, and LP interpreted the results and wrote the manuscript. All authors read and approved the final manuscript.

\section{Funding}

GG, VS, and LP were partially funded by NIH U19MH114830.

\section{Availability of data and materials}

CITE-seq RNA and protein data were acquired from Gene Expression Omnibus samples GSM2695381 and GSM2695382 [31]. REAP-seq RNA and protein data were acquired from GSM2685238 and GSM2685243 [32]. ECCITE-seq control protein data were acquired from GSM3596096 [33]. ECCITE-seq CTCL protein data were acquired from GSM3596101 [33]. Due to patient privacy concerns, raw ECCITE-seq RNA data (GSM3596095 and GSM3596100) were not available, and the gene count matrices generated by velocyto were acquired by personal request. 10X Genomics $1 \mathrm{k}$ and 10k PBMC datasets were acquired from the 10X Genomics website [20, 21].

The datasets generated during this study are available on figshare [2730].The Jupyter scripts used to analyze them are available on GitHub [26]. The protaccel Python package is available for installation through PyPi [24], and may be acquired as a script from GitHub [26] or Zenodo [34] under the BSD-2-Clause license.

\section{Ethics approval and consent to participate}

Not applicable.

\section{Consent for publication}

Not applicable.

\section{Competing interests}

The authors declare that they have no competing interests.

\section{Author details}

${ }^{1}$ Division of Chemistry and Chemical Engineering, California Institute of Technology, Pasadena, USA. ²Division of Biology and Biological Engineering $\&$ Department of Computing and Mathematical Sciences, California Institute of Technology, Pasadena, USA.

Received: 9 July 2019 Accepted: 24 January 2020 Published online: 18 February 2020

\section{References}

1. Svensson V, Vento-Tormo R, Teichmann SA. Exponential scaling of singlecell RNA-seq in the past decade. Nat Protoc. 2018;13(4):599-604.

2. Stuart T, Satija R. Integrative single-cell analysis. Nat Rev Genet. 2019;20(5): 257-72.

3. Stoeckius M, Hafemeister C, Stephenson W, Houck-Loomis B, Chattopadhyay PK, Swerdlow $\mathrm{H}$, et al. Simultaneous epitope and transcriptome measurement in single cells. Nat Methods. 2017;14(9):865-8.

4. Peterson VM, Zhang KX, Kumar N, Wong J, Li L, Wilson DC, et al. Multiplexed quantification of proteins and transcripts in single cells. Nat Biotechnol. 2017;35(10):936-9.

5. Mimitou EP, Cheng A, Montalbano A, Hao S, Stoeckius M, Legut M, et al. Multiplexed detection of proteins, transcriptomes, clonotypes and CRISPR perturbations in single cells. Nat Methods. 2019;16(5):409-12.

6. La Manno G, Soldatov R, Zeisel A, Braun E, Hochgerner H, Petukhov V, et al. RNA velocity of single cells. Nature. 2018;560(7719):494-8.

7. Zeisel A, Kostler WJ, Molotski N, Tsai JM, Krauthgamer R, Jacob-Hirsch J, et al. Coupled pre-mRNA and mRNA dynamics unveil operational strategies underlying transcriptional responses to stimuli. Mol Syst Biol. 2014;7(1):529.

8. Svensson V, Pachter L. RNA velocity: molecular kinetics from single-cell RNASeq. Mol Cell. 2018;72(1):7-9.

9. Munsky B, Trinh B, Khammash M. Listening to the noise: random fluctuations reveal gene network parameters. Mol Syst Biol. 2009;5:318.

10. Schnoerr D, Sanguinetti G, Grima R. Approximation and inference methods for stochastic biochemical kinetics - a tutorial review. J Phys A Math Theor. 2017;50(9):093001.
11. Golan-Lavi R, Giacomelli C, Fuks G, Zeisel A, Sonntag J, Sinha S, et al. Coordinated pulses of mRNA and of protein translation or degradation produce EGF-induced protein bursts. Cell Rep. 2017;18(13):3129-42.

12. Mauri C, Bosma A. Immune regulatory function of B cells. Annu Rev Immunol. 2012;30(1):221-41.

13. Parker DC. T cell-dependent B cell activation. Annu Rev Immunol. 1993; 11(1):331-60.

14. Rosser EC, Mauri C. Regulatory B cells: origin, phenotype, and function. Immunity. 2015;42(4):607-12.

15. Ricciardi S, Manfrini N, Alfieri R, Calamita P, Crosti MC, Gallo S, et al. The translational machinery of human CD4+ T cells is poised for activation and controls the switch from quiescence to metabolic remodeling. Cell Metabolism. 2018;28(6):895-906 e5.

16. O'Shea JJ, Paul WE. Mechanisms underlying lineage commitment and plasticity of helper CD4+ T cells. Science. 2010;327(5969):1098-102.

17. Jakubzick CV, Randolph GJ, Henson PM. Monocyte differentiation and antigen-presenting functions. Nat Rev Immunol. 2017;17(6):349-62.

18. Narasimhan PB, Marcovecchio P, Hamers AAJ, Hedrick CC. Nonclassical monocytes in health and disease. Annu Rev Immunol. 2019;37(1):439-56.

19. Qiu X, Zhang Y, Yang D, Hosseinzadeh S, Wang L, Yuan R, et al. Mapping Vector Field of Single Cells. Biorxiv. 2019;9:696724.

20. $1 \mathrm{k}$ PBMCs from a Healthy Donor - Gene Expression and Cell Surface Protein. 10X Genomics [cited 2019 Sept 17]. Available from: https://support.1 0xgenomics.com/single-cell-gene-expression/datasets/3.0.0/pbmc_1k_ protein_v3.

21. 10k PBMCs from a Healthy Donor - Gene Expression and Cell Surface Protein. 10X Genomics [cited 2019 Sept 17]. Available from: https:// support.10xgenomics.com/single-cell-gene-expression/datasets/3.0.0/ pbmc_10k_protein_v3 .

22. Pedregosa F, Varoquaux G, Gramfort A, Michel V, Thirion B, Grisel O, et al. Scikit-learn: machine learning in Python. J Mach Learn Res. 2011;12:2825-30.

23. Blondel VD, Guillaume J-L, Lambiotte R, Lefebvre E. Fast unfolding of communities in large networks. J Stat Mech 2008;2008(10):P10008.

24. Gorin G. protaccel. PyPi; 2019 [cited 2019 Dec 17]. Available from: https:// pypi.org/project/protaccel/ .

25. van der Maaten L, Hinton G. Visualizing data using t-SNE. J Mach Learn Res. 2008;9:2579-605.

26. Gorin G, Svensson V, Pachter L. Protein acceleration Github repository. Github; 2020 [cited 2019 Dec 17]. Available from: https://github.com/ pachterlab/GSP_2019

27. Gorin G, Svensson V, Pachter L. CITE-seq protein and mRNA counts. figshare 2019 [cited 2020 Jan 20]. Available from: https:/figshare.com/articles/CITEseq_protein_and_mRNA_counts/8309696

28. Gorin G, Svensson V, Pachter L. REAP-seq protein and mRNA counts. figshare; 2019 [cited 2020 Jan 20]. Available from: https://figshare.com/ articles/REAP-seq_protein_and_mRNA_counts/8309708

29. Gorin G, Svensson V, Pachter L. ECCITE-seq protein and mRNA counts. figshare; 2019 [cited 2020 Jan 20]. Available from: https:/figshare.com/ articles/ECCITE-seq_protein_and_mRNA_counts/8309714

30. Gorin G, Pachter L, Svensson V. 10X protein and mRNA counts. figshare; 2019 [cited 2020 Jan 20]. Available from: https://figshare.com/articles/10X_ protein_and_mRNA_counts/9912734/1

31. Stoeckius M. CITE-seq: large scale simultaneous measurement of epitopes and transcriptomes in single cells [Internet]. NCBI Gene Expression Omnibus. 2017 [cited 2018 Oct 22]. Available from: https:/www.ncbi.nlm.nih.gov/geo/query/acc.cgi?acc= GSE100866.

32. Peterson VM, Zhang KX. The dynamics of cellular response to therapeutic perturbation using multiplexed quantification of the proteome and transcriptome at single-cell resolution [Internet]. NCBI Gene Expression Omnibus. 2017 [cited 2018 Nov 19]. Available from: https://www.ncbi.nlm. nih.gov/geo/query/acc.cgi?acc=GSE100501

33. Mimitou EP, Smibert P. Expanding the CITE-seq tool-kit: detection of proteins, transcriptomes, clonotypes and CRISPR perturbations with multiplexing, in a single assay [Internet]. NCBI Gene Expression Omnibus. 2019 [cited 2019 Apr 29]. Available from: https:/uww.ncbi.nlm.nih.gov/geo/query/acc.cgi?acc=GSE126310.

34. Gorin G, Svensson V, Pachter L. protaccel Python package [Internet]. Zenodo; 2019 [cited 2019 Dec 17]. Available from: https://doi.org/10.5281/ zenodo.3620874.

\section{Publisher's Note}

Springer Nature remains neutral with regard to jurisdictional claims in published maps and institutional affiliations. 